\title{
Experimentally obtained velocity and pressure fields of an open channel flow around a cylinder using RIM-SPIV
}

\author{
W. J. Reeder ${ }^{1 *}$, J. R. Moreto ${ }^{2}$, X. Liu ${ }^{2}$, D. Tonina ${ }^{1}$ \\ ${ }^{1}$ Center for Ecohydraulics Research, University of Idaho, Boise, U.S.A \\ ${ }^{2}$ Department of Aerospace Engineering, San Diego State University, San Diego, California, U.S.A. \\ *wjreeder@uidaho.edu
}

\begin{abstract}
The quantification of velocity and pressure fields over streambeds is important for predicting sediment mobility and water exchange between stream and sediment interstitial spaces (Schmeeckle and Nelson, 2003, Tonina and Buffington, 2009). The latter is typically referred as hyporheic flow, which consists of surface water that flows through the streambed sediment pores (Tonina and Buffington, 2009). These fluxes are mainly driven by pressure gradients at the water sediment interface. In this paper, we report an experimental investigation of the time-averaged velocity and pressure field, quantified in a set of laboratory experiments using stereo PIV (Particle Image Velocimetry) with a non-toxic index-matched fluid, for an open channel flow around a barely submerged vertical cylinder as a model for plant stalk over a plane bed of coarse granular sediment, mimicking a stream gravel bed. This is the first time that such a velocity and pressure field is characterized experimentally for a free surface flow with irregular floor contour.
\end{abstract}

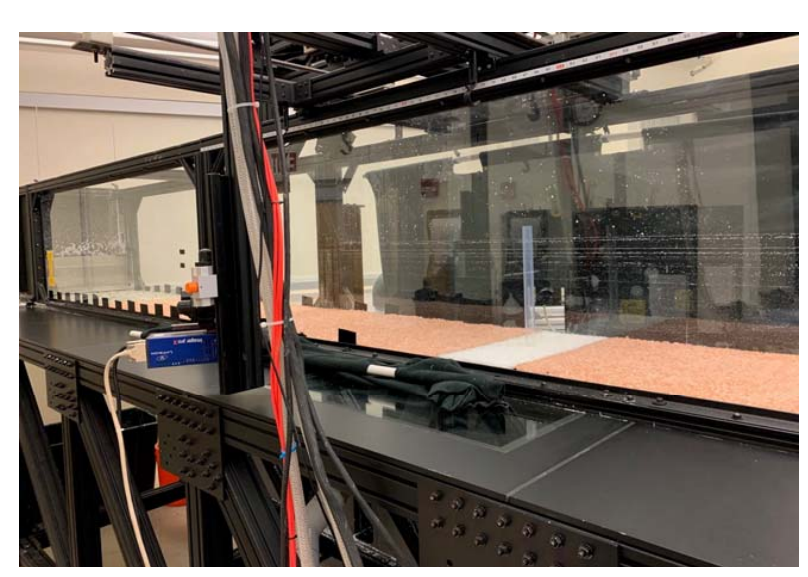

(a)

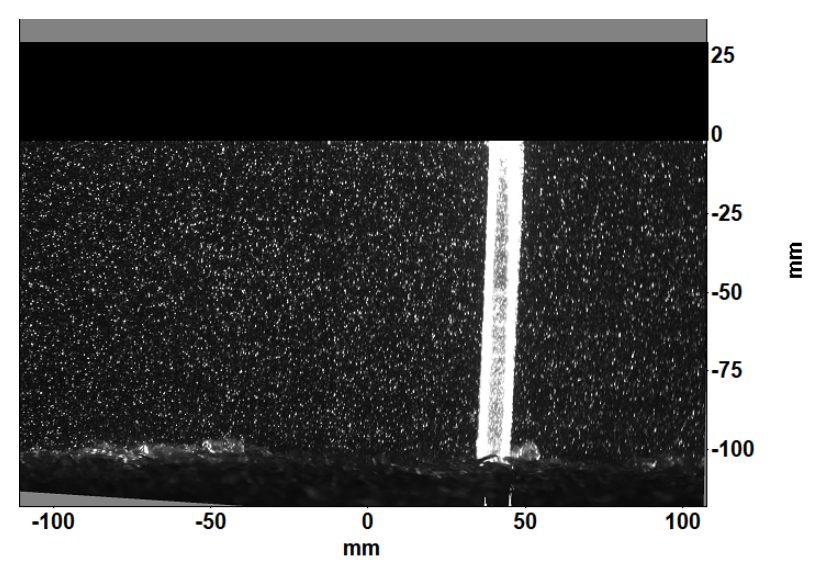

(b)

Figure 1: Experimental set up (left) with vertical cylinder and the distortion corrected SPIV image (right).

The near-bed pressure distribution can be complex and difficult to measure with direct techniques such as piezometers, pressure transducers or Pitot tubes because of small magnitude variations riding on top of a large hydrostatic pressure field. This is true in both field and laboratory setting. In the latter case, recent advances in stereoscopic particle imaging velocimetry, SPIV, allow detailed and high-resolution measurements of the flow field including turbulent fluctuations (De Kat and Van Oudheusden, 2012; van Oudheusden, 2013) from which the pressure field can be quantified by knowing the pressure at one location. SPIV has the advantage of being non-intrusive and providing the three velocity components along with accurate quantification of their uncertainties. However, being an optical method, it relies on "seeing" the flow within the entire area of interest. This is not possible in case of solid objects immersed in the fluid because they can 
cause shadows or partially/fully block the view of the flow. To avoid this problem, laboratory experiments use refractive index matching (RIM) techniques (Budwig, 1994), in which both solid and fluid exhibit similar optical properties, such that they are virtually indistinguishable (Figure 1), i.e., the solid is functionally transparent, and SPIV can see through the solid and map the flow field all around it. Application of this technique is typically expensive and/or hazardous, because of the use of specialized and toxic liquids coupled with commonly used transparent solid materials, typically glass (Budwig, 1994). The pressure field is then reconstructed from SPIV velocity data by first locally applying the momentum (Navier-Stokes) equations in differential form and successively integrating the pressure gradient to obtain the pressure field (De Kat and Van Oudheusden, 2012; Liu and Katz, 2006; van Oudheusden, 2013).

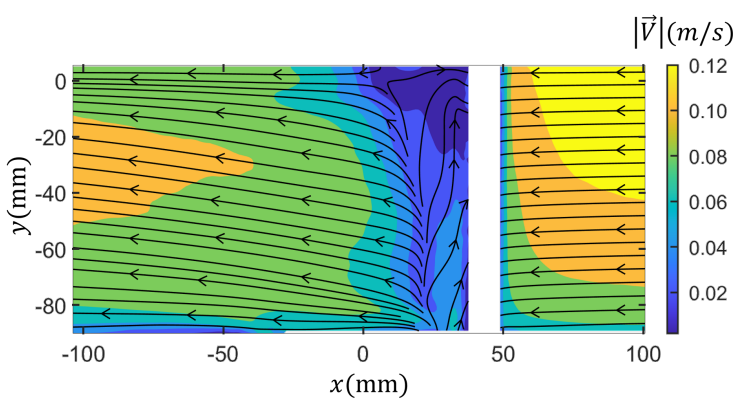

(a)

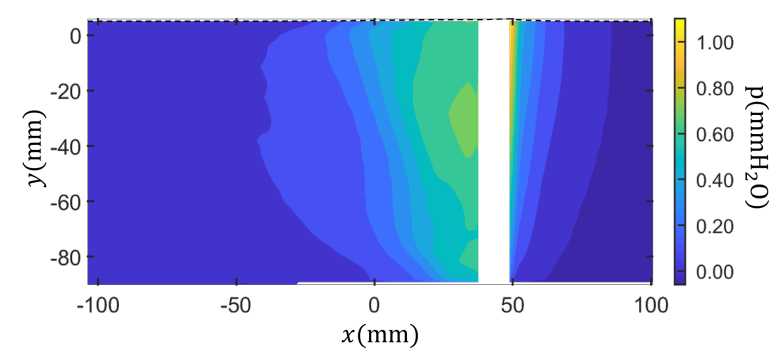

(b)

Figure 2: Sample mean velocity (left) and mean pressure (right, without accounting the body force) along the symmetry plane through the center of the cylinder. Please note the flow direction is from right to left.

This pressure reconstruction has been shown to be effective in several previous works (Joshi et al., 2014, De Kat and Van Oudheusden, 2012; Liu and Katz, 2006, 2008, 2013; Liu and Moreto, 2020; Liu et al., 2016). However, these applications were based on geometries with smooth boundaries, well-defined location of the water surface elevation and without large solid bodies protruding within the flow. However, such protrusions are typical in experimental studies of flows over coarse sediments with large macro-roughness elements, such as boulders, bedforms and aquatic vegetation, which depending on their submergence may cause complex water surface elevation patterns. Here, we investigate the velocity and pressure fields around a submerged vertical cylinder, which mimics a single rigid stem of aquatic vegetation over a rough bed. The experiment was in a $7 \mathrm{~m}$ long and $0.5 \mathrm{~m}$ wide flume with glass wall (Figure 1(a)). The flume bottom was covered with granular material made of crashed glass with mean diameter of $2.5 \mathrm{~mm}$ arranged to mimic a plane bed. The cylinder was placed in the center of the flume and made of a transparent polymer with specific gravity of nearly 2 and refractive index of 1.36 similar to that of water (1.33). To match the solid refractive index, we added $15 \%$ in weight of Epsom salt to the water. This fluid mixture is non-toxic, with density $1,158 \mathrm{~kg} / \mathrm{m}^{3}$ and dynamic viscosity $2.9710^{-3} \mathrm{~kg} /(\mathrm{m} \cdot \mathrm{s})$. It is relatively inexpensive and allows using refractive index matching (RIM) technique (Figure $1(\mathrm{~b})$ ).

We adopted coupled RIM and SPIV to measure the flow field simultaneously upstream and downstream the cylinder along its center (Figure 2(a)). The cylinder with a $1.0 \mathrm{~cm}$ diameter was $10 \mathrm{~cm}$ tall and the flow field had a mean hydraulic depth of approximately $10.1 \mathrm{~cm}$ and mean velocity of approximately $0.12 \mathrm{~m} / \mathrm{s}$.

We reconstructed the pressure field around the cylinder over the rough bed by using the parallel-ray omni-directional algorithm from the measured flow field (Liu and Moreto, 2020; Liu et al., 2016). To accommodate the constant pressure along the free surface and the irregular shape of the channel bed, appropriate adaptation of boundary treatment for the pressure reconstruction code were implemented. The use of RIM-PIV allowed us to map the flow hydraulics continuously around the cylinder. The method shows that mapping of the hydrodynamic fields around solids can be achieved experimentally with the use of low-cost and non-toxic fluids or solids coupled with suitable pressure field reconstructing algorithms. The analysis presented here can be extended to study flow and pressure fields around and within solids for various hydraulic applications.

\section{Acknowledgements}

This project is partially funded by the National Science Foundation under award number EAR1559348, 
USDA National Institute of Food and Agriculture, Hatch project 1012806 and the San Diego State University.

\section{References}

Budwig R (1994) Refractive index matching methods for liquid flow investigations. Experiments in Fluids $17: 350-355$

De Kat R and Van Oudheusden BW (2012) Instantaneous planar pressure determination from PIV in turbulent flow. Experiments in Fluids 52:1089-1106

Joshi P, Liu X, and Katz J (2014) Effect of mean and fluctuating pressure gradients on boundary layer turbulence. Journal of Fluid Mechanics 748:36-84

Liu X and Katz J (2006) Instantaneous pressure and material acceleration measurements using a four exposure PIV system. Experiments in Fluids 41:227-240

Liu X and Katz J (2008) Cavitation phenomena occurring due to interaction of shear layer vortices with the trailing corner of a two-dimensional open cavity. Physics of Fluids 20:041702

Liu X and Katz J (2013) Vortex-corner interactions in a cavity shear layer elucidated by time-resolved measurements of the pressure field. Journal of Fluid Mechanics 728:417-457

Liu X and Moreto JR (2020) Error propagation from the PIV-based pressure gradient to the integrated pressure by the omnidirectional integration method. Measurement Science and Technology 31:055301

Liu X, Moreto JR, and Siddle-Mitchell S (2016) Instantaneous Pressure Reconstruction from Measured Pressure Gradient using Rotating Parallel Ray Method. in 54th AIAA Aerospace Sciences Meeting. volume 0. pages 1-8. American Institute of Aeronautics and Astronautics, Reston, Virginia

Schmeeckle MW and Nelson JM (2003) Direct numerical simulation of bedload transport using a local, dynamic boundary condition. Sedimentology 50:279-301

Tonina D and Buffington JM (2009) Hyporheic exchange in mountain rivers I: Mechanics and environmental effects. Geography Compass 3:1063-1086

van Oudheusden BW (2013) PIV-based pressure measurement. Measurement Science and Technology 24:032001 